\title{
THE DISTRIBUTION OF FUNCTIONAL OF A TRAJECTORY OF A PARTICLE EXECUTING A RANDOM WALK IN A DISORDERED MEDIUM
}

\author{
V.P. Shkilev, V.V. Lobanov \\ Chuiko Institute of Surface Chemistry, National Academy of Sciences of Ukraine \\ 17 General Naumov Str., Kyiv, 03164,Ukraine, e-mail:lobanov@isc.gov.ua
}

The problem of finding the distribution of functional of a trajectory of a particle executing a random walk in a disordered medium containing both traps and obstacles is considered. As a model of a disordered medium, the Schirmacher model, which is the combination of the random barriers model and the multiple-trapping model, is used. Forward and backward Feynman-Kac equations with the boundary conditions at discontinuity points are formulated. As an example, the distribution of the residence time in a half-space is obtained. It is shown that the anomalous subdiffusion due to traps and that due to obstacles give very different distributions.

\section{Introduction}

1.1. Functional of a random-walk trajectory

A Brownian functional is defined as

$$
A=\int_{0}^{t} U(x(\tau)) \mathrm{d} \tau
$$

where $x(\tau)$ is a trajectory of a Brownian particle and $U(x)$ is a prescribed function, the type of which depends on the problem considered. Brownian functionals arise in various fields of science; for example, a functional equal to the time spent by the particle in a given domain arises in chemical kinetics $[1,2,6,8]$. In this case, $U(x)=1$ in domain and $U(x)=0$ otherwise. Other examples include functionals with functions $U(x)=x$ and $U(x)=x^{2}$, which are of interest for the theory of nuclear magnetic resonance [9]: the case $U(x)=x^{2}$ was considered in the study of the dynamics of the growing surface [5].

Using the path integral method proposed by Feynman, Kac derived a partial differential equation which allows one to find the distribution of the Brownian functional with an arbitrary positive function $U(x)$ [11]:

$$
\frac{\partial G(x, p, t)}{\partial t}=D \frac{\partial^{2} G(x, p, t)}{\partial x^{2}}-p U(x) G(x, p, t) .
$$

Here $G(x, p, t)$ is the Laplace transform in variable $A$ for the function $G(x, A, t)$ equal to the joint probability that, at time $t$, the particle is at point $x$ and the functional is equal to $A ; D$ is the diffusion coefficient. This Feynman-Kac equation has been widely used for the calculation of distributions of functional with different functions $U(x)$ [14].

Equation (1.2) was derived under the assumption that the medium in which the process occurs is homogeneous. If the medium is inhomogeneous, the calculation of the distribution of the functional becomes much more complicated $[13,17]$; however, if the selfaveraging property holds, then for an inhomogeneous medium we can obtain an equation similar to equation (1.2). In [19], such equations (forward and backward) were derived for media that can be described by the random traps model. In this model, the diffusion-slowing occurs due to the particle delay in the traps. In the present paper, equations are derived in a 
more realistic model which takes into account the presence of both traps and different kinds of obstacles in a disordered medium. In such a case, other equations are obtained, because obstacles create an essentially new mechanism for diffusion-slowing. This mechanism consists in the fact that the particle cannot move with equal probability in all directions, so that the negative velocity correlations arise.

Interest in the study of functional of a random-walk trajectory has increased with the development measurement techniques. The latest methods allow one to track the trajectory of the individual particles, and thus to find the distribution of different functional experimentally. By analyzing these distributions, one can obtain valuable information about the characteristics of random walks; particular, by the structure of environment in which they occur. To do this, we need appropriate theoretical models.

For the first time, the generalized Feynman-Kac equations were derived in $[4,22]$ in the framework of the continuous time random walk model. In principle, these equations can be used to find the distributions of functionals in disordered media; however, they, as well as the random traps equations, take into account only one diffusion-slowing mechanism - the delay in the traps. Generalized Feynman-Kac equations were recently derived in [3] on the basis of the Langevin equations. Since this approach assumes that the particle can move with equal probability in any direction, the resulting equations are also equivalent to the random traps equations.

We are interested in calculating the distribution of the functional (1.1) when the motion of a particle whose trajectory appears under the integral is described by the equation

$$
\frac{\partial P_{n}(t)}{\partial t}=\sum_{m} W_{n m} P_{m}(t)-\sum_{m} W_{m n} P_{n}(t)
$$

where $P_{n}(t)$ is the probability that the particle is in site $n$ at time $t$ and $W_{n m}$ is the transition rate from site $m$ to site $n$. In [19], it was shown that at any structure of the lattice, i.e. for any parameters $W_{n m}$, the joint distribution of the functional (1.1) and the particle coordinates satisfies the equation

$$
\frac{\partial G_{n}(p, t)}{\partial t}=\sum_{m} W_{n m} G_{m}(p, t)-\sum_{m} W_{m n} G_{n}(p, t)-p U_{n} G_{n}(p, t) .
$$

Here $G_{n}(p, t)$ is Laplace transform in variable $A$ for the function $G_{n}(A, t)$ equal to the joint probability that the particle is at site $n$ at time $t$ and the functional is equal to $A ; U_{n}$ is value of $U(x)$ at the site $n$. This equation describes a random walk with a first order chemical reaction whose rate $p U_{n}$ varies in space. The variable $p$ plays the role of a parameter.

If the self-averaging property holds, then the averaged over elementary physical volume distribution of the functional, for any extended sample of a disordered medium at large times will coincide with the distribution found by solving equation (1.4) and averaging this solution over the ensemble of configurations. Thus, if the self-averaging property holds, the problem reduces to finding the averaged over the ensemble of configurations solutions of equation (1.4). In this paper we obtain the equations which this averaged solutions must satisfy within the Schirmacher model $[7,15,18]$.

\subsection{Schirmacher model}

In the Schirmacher model, all lattice sites are divided into two types: transport states among which hopping is allowed and traps which are only accessible via the transport states. The equation (1.3) takes the form 


$$
\begin{aligned}
& \frac{\partial P_{n}(t)}{\partial t}=\sum_{m} \omega_{n m} P_{m}(t)-\sum_{m} \omega_{m n} P_{n}(t)-\kappa_{n} P_{n}(t)+\nu_{n} Q_{n}(t), \\
& \frac{\partial Q_{n}(t)}{\partial t}=\kappa_{n} P_{n}(t)-\nu_{n} Q_{n}(t)
\end{aligned}
$$

where $P_{n}(t)$ is the probability that the particle is in transport state $n$ at time $t ; Q_{n}(t)$ is the probability that the particle is in trap $n$ at time $t ; \omega_{m n}$ is the transition rate from transport state $n$ to transport state $m ; \kappa_{n}$ and $v_{n}$ are, respectively, the transitions rates from the $n$-th transport state to the $n$-th trap and back. As a result of averaging of this equations over the ensemble of configurations and passing to the continuum limit, the authors of $[7,15,18]$ obtained following equation for the averaged probability density of finding a particle at the point $x$ at the time $t \rho(x, t)$ :

$$
\frac{\partial \rho(x, t)}{\partial t}=a^{2} \int_{0}^{t} \Theta(t-\tau) \frac{\partial^{2}}{\partial x^{2}} \rho(x, \tau) \mathrm{d} \tau .
$$

Here $a^{2}$ is the parameter and $\Theta(t)$ is the memory function that in Laplace space $t \rightarrow s$ can be represented as $\Theta(s)=\Psi(s) \Phi(s)$, where

$$
\begin{aligned}
& \Psi(s)=\Lambda(s+\Sigma(s)), \\
& \Phi(s)=s /(s+\Sigma(s)) .
\end{aligned}
$$

Here $\Lambda(s)$ is the memory function that can be obtained by averaging equation (1.5) at zero parameters $\kappa_{n}$ and $v_{n}$, i.e., in the absence of traps and $\Sigma(s)$ is the function that describes the effect of traps on dispersive (anomalous) transport. These functions can be calculated in different approximations. The works [7, 15, 18], proposed methods for finding functions $\Lambda(s)$ in EMA (effective medium approximation) and $\Sigma(s)$ in CPA (coherent potential approximation). In this paper, specific forms of these functions will not be used.

In [21], the Schirmacher model was generalized to the case when the diffusing particle could disappear according to the first order chemical reaction with space dependent reaction rate $k_{n}$. The terms $-k_{n} P_{n}(t)$ and $-k_{n} Q_{n}(t)$ were added to the right sides of equations (1.5) and (1.6), respectively. It was assumed that the motion through the transport state described by the random barriers model, i.e., that the transport transition rates are symmetric $\left(\omega_{m n}=\omega_{n m}\right)$. After averaging and passing to the continuum limit, the following equation was obtained:

$$
\frac{\partial \rho(x, t)}{\partial t}=a^{2} \frac{\partial}{\partial x} \int_{0}^{t}\left\{\Psi^{*}(x, t-\tau) \frac{\partial}{\partial x} \int_{0}^{\tau} \Phi^{*}(x, \tau-\zeta) \rho(x, \zeta) \mathrm{d} \zeta\right\} \mathrm{d} \tau-k(x) \rho(x, t),
$$

where $k(x)$ is the reaction rate constant (a continuum extension of the $k_{n}$ );

$$
\begin{aligned}
& \Psi^{*}(x, t)=\Psi(t) \exp (-k(x) t), \\
& \Phi^{*}(x, t)=\Phi(t) \exp (-k(x) t) .
\end{aligned}
$$

The functions $\Psi(t)$ and $\Phi(t)$ enter into equation (1.10) separately and not as the combination $\Theta(t)=\int_{0}^{t} \Psi(t-\tau) \Phi(\tau) d \tau$ as in the case of equation (1.7). Therefore, this equation will have different forms depending on contributions made by barriers and by traps to the dispersive 
transport. For example, if dispersive transport is caused by barriers without traps $\left(\sum(s)=0\right)$, the equation would look like

$$
\frac{\partial \rho(x, t)}{\partial t}=a^{2} \frac{\partial}{\partial x} \int_{0}^{t} \Theta^{*}(x, t-\tau) \frac{\partial}{\partial x} \rho(x, \tau) \mathrm{d} \tau-k(x) \rho(x, t),
$$

and if it is caused by traps without barriers $(\Lambda(s)=$ const $)$, then the equation would be

$$
\frac{\partial \rho(x, t)}{\partial t}=a^{2} \frac{\partial^{2}}{\partial x^{2}} \int_{0}^{t} \theta^{*}(x, t-\tau) \rho(x, \tau) d \tau-k(x) \rho(x, t),
$$

where $\Theta^{*}(x, t)=\Theta(t) \exp (-k(x) t)$. The solutions of the equations (1.13) and (1.14) with the same function $\Theta^{*}(x, t)$ may differ materially [21]. This means that the barriers and traps manifest themselves differently in the diffusion-reaction processes, if a chemical reaction constant varies in space. This fact, in principle, can be used to determine the microscopic structure of a disordered medium.

\section{Forward equation}

2.1. Particle is in transport state at the initial time

In the Shirmacher model, the equation (1.4) takes the form

$$
\begin{aligned}
& \frac{\partial F_{n}(p, t)}{\partial t}=\sum_{m} \omega_{m m} F_{m}(p, t)-\sum_{m} \omega_{m n} F_{n}(p, t)-\kappa_{n} F_{n}(p, t)+\nu_{n} C_{n}(p, t)-p U_{n} F_{n}(p, t), \\
& \frac{\partial C_{n}(p, t)}{\partial t}=\kappa_{n} F_{n}(p, t)-\nu_{n} C_{n}(p, t)-p U_{n} C_{n}(p, t) .
\end{aligned}
$$

Functions $F_{n}(p, t)$ and $C_{n}(p, t)$ are the parts of the function $C_{n}(p, t)$ which correspond to the position of the particle in the transport state and trap, respectively. From the above it follows that within the Shirmacher model, averaged distribution $G(x, p, t)$ must satisfy the equation, differs from equation (1.10) only in the form of reaction constant:

$$
\frac{\partial G(x, p, t)}{\partial t}=a^{2} \frac{\partial}{\partial x} \int_{0}^{t}\left\{\Psi^{*}(x, t-\tau) \frac{\partial}{\partial x} \int_{0}^{\tau} \Phi^{*}(x, \tau-\zeta) G(x, p, \zeta) \mathrm{d} \zeta\right\} \mathrm{d} \tau-p U(x) G(x, p, t),
$$

where

$$
\begin{aligned}
& \Psi^{*}(x, t)=\Psi(t) \exp (-p U(x) t), \\
& \Phi^{*}(x, t)=\Phi(t) \exp (-p U(x) t) .
\end{aligned}
$$

This equation is a direct Feynman-Kac equation for the considered model. It differs from the analogous equation of the random traps model and equations obtained in [4, 22], by the presence of an additional memory function $\Psi^{*}(x, t)$ caused by barriers.

Function $G(x, p, t)$ gives the joint distribution of the functional (1.1) and coordinate of the particle at time $t$. To find the distribution of functional only, we need to integrate this function in the coordinate $x$. In particular, to find $H\left(x_{0}, A, t\right)$ - the distribution of functional (1.1) corresponding to the starting point $x_{0}$, it is necessary to find a solution of equation (2.3), corresponding to the initial condition $G(x, p, 0)=\delta\left(x-x_{0}\right)$ (we denote it by $G\left(x, p, t ; x_{0}\right)$ intedrating this solution with respect to $x$ : 


$$
H\left(x_{0}, p, t\right)=\int_{-\infty}^{\infty} G\left(x, p, t ; x_{0}\right) \mathrm{d} x,
$$

and taking the inverse Laplace transform $p \rightarrow A$.

\subsection{Particle is not necessary in transport state at the initial time}

Equation (2.3) is obtained under the assumption that the particle is in the transport state at the initial time $[7,15,18,21]$. However, this assumption is not always satisfied. In some cases, the experiment is carried out so that the probability distribution of finding a particle in different states is equilibrium. In this section, we derive a generalization of (2.3) in case of arbitrary distribution.

First, consider the case when there is no chemical reaction. Suppose that at the initial time the probability of finding the particle in the trap of $i$-th type is $\theta_{i}^{0}$. Then the probability that the particle will remain trapped until the time $t$ is equal to

$$
\sum_{i=1}^{N} \theta_{i}^{0} \exp \left(-\nu_{i} t\right)
$$

and the probability that the particle will make first transition from a trap to the transport state in the interval $(t, t+d t)$ is equal to

$$
\sum_{i=1}^{N} \theta_{i}^{0} \nu_{i} \exp \left(-\nu_{i} t\right) d t
$$

where $v_{i}$ is the transitions rate from the trap of $i$-th type to the transport state and $N$ is the number of traps types. Using these expressions, we can write the equation for the probability of finding the particle in the transport state.

In the Schirmacher model, the total probability of finding the particle at the point $x$, $\rho(x, t)$ and the probability $P(x, t)$ - of finding the particle in the transport state at same point, are related by (in Laplace domain $t \rightarrow s$ )

$$
\rho(x, s)=P(x, s)+(\Sigma(s) / s) P(x, s) .
$$

Here, the second term on the right represents the probability that the particle is trapped. Equation (1.7) in terms of the function $P(x, s)$ has the form

$$
s P(x, s)-\delta\left(x-x_{0}\right)=a^{2} \Psi(s) \frac{\partial^{2}}{\partial x^{2}} P(x, s)-\Sigma(s) P(x, s) .
$$

This equation is valid if, at the initial time the particle is in the transport state. If there is a nonzero probability that the particle is trapped, then the equation is as follows:

$$
s P(x, s)-P_{0} \delta\left(x-x_{0}\right)=a^{2} \Psi(s) \frac{\partial^{2}}{\partial x^{2}} P(x, s)-\Sigma(s) P(x, s)+\sum_{i=1}^{N} \frac{\theta_{i}^{0} \nu_{i}}{s+\nu_{i}} \delta\left(x-x_{0}\right),
$$

where $P_{0}$ is the probability that a particle is in the transport state at the initial time: $P_{0}=1-\sum_{i=1}^{N} \theta_{i}^{0}$. The last term in the right-hand side describes the arrival of the particles into the transport state, represented by the formula (2.8). In this case, the total probability is expressed as 


$$
\rho(x, s)=P(x, s)+(\Sigma(s) / s) P(x, s)+\sum_{i=1}^{N} \frac{\theta_{i}^{0}}{s+\nu_{i}} \delta\left(x-x_{0}\right) .
$$

Here, the second term on the right represents the probability that the particle is trapped, but before it has already been in the transport state. The third term represents the probability that the particle is trapped without having visited the transport state; this term corresponds to the probability (2.7). The equation for the overall probability is written as

$$
s \rho(x, s)-\delta\left(x-x_{0}\right)=a^{2} \Theta(s) \frac{\partial^{2}}{\partial x^{2}} \rho(x, s)-a^{2} \Theta(s) \sum_{i=1}^{N} \frac{\theta_{i}^{0}}{s+\nu_{i}} \frac{\partial^{2}}{\partial x^{2}} \delta\left(x-x_{0}\right) .
$$

If, at the initial time, the probability is not concentrated at one point, and distributed over a certain region, the last term in this equation is of the form $a^{2} \Theta(s) \sum_{i=1}^{N} \frac{\partial^{2}}{\partial x^{2}} \frac{\theta_{i}^{0}(x)}{s+v_{i}}$. The consequence of (2.13) is that the resulting flux in the considered model is given by

$$
J(x, s)=-a^{2} \Theta(s) \frac{\partial}{\partial x}\left\{\rho(x, s)-\sum_{i=1}^{N} \frac{\theta_{i}^{0}(x)}{s+\nu_{i}}\right\} .
$$

Using this expression and the approach proposed in [21], we can generalize the equation (2.13) for the case when the diffusing particle disappears according to the reaction of the first order. As a result, we obtain the equation

$$
\begin{aligned}
& \frac{\partial \rho(x, t)}{\partial t}=a^{2} \frac{\partial}{\partial x} \int_{0}^{t}\left\{\Psi^{*}(x, t-\tau) \frac{\partial}{\partial x} \int_{0}^{\tau} \Phi^{*}(x, \tau-\zeta) \rho(x, \zeta) \mathrm{d} \zeta\right\} \mathrm{d} \tau- \\
& -a^{2} \frac{\partial}{\partial x} \int_{0}^{t}\left\{\Psi^{*}(x, t-\tau) \frac{\partial}{\partial x} \int_{0}^{\tau} \Omega^{*}(x, \tau)\right\} \mathrm{d} \tau-k(x) \rho(x, t),
\end{aligned}
$$

where $\Omega^{*}(x, t)=\Omega(t) \exp (-k(x) t) ; \Omega(t)$ is the function that in Laplace domain $t \rightarrow s$ can be represented as

$$
\Omega(s)=\Phi(s) \sum_{i=1}^{N} \frac{\theta_{i}^{0}(x)}{s+\nu_{i}} .
$$

Forward Feynman-Kac equation is obtained from (2.15) by substituting $G(x, p, t)$ instead of $\rho(x, t)$ and $p U(x)$ instead of $k(x)$. The solution of the obtained equation corresponding to starting point $x_{0}$ can be represented as the sum of regular and singular terms. In Laplace domain $t \rightarrow s, A \rightarrow p$ we will have

$$
G\left(x, p, t ; x_{0}\right)=G_{r}\left(x, p, s ; x_{0}\right)+\frac{1-\phi(s+p U(x))}{s+p U(x)} \delta\left(x-x_{0}\right),
$$

where the $G\left(x, p, s ; x_{0}\right)$ satisfies the equation

$$
\begin{aligned}
& {[s+p U(x)] G_{r}\left(x, p, s ; x_{0}\right)-\phi(s+p U(x)) \delta\left(x-x_{0}\right)=} \\
& =a^{2} \frac{\partial}{\partial x}\left\{\Psi(s+p U(x)) \frac{\partial}{\partial x}\left[\Phi(s+p U(x)) G_{r}\left(x, p, s ; x_{0}\right)\right]\right\} .
\end{aligned}
$$


Here

$$
\Omega(s)=\Phi(s) \sum_{i=1}^{N} \frac{\theta_{i}^{0}(x)}{s+\nu_{i}} .
$$

\section{Boundary conditions}

The function $U(x)$ can have discontinuities at certain points. In order for the properties of the original discrete model to reflect correctly by a continuous model, it is necessary to put the correct boundary conditions at these points. In this section, the boundary conditions for the equation (2.18) at a points of discontinuity of the function $U(x)$ are considered on a simple example of a regular one-dimensional lattice. A more general approach to the problem of boundary conditions is provided in [20].

First, consider the case where there are no traps: $\kappa_{n}=0, v_{n}=0$. In one-dimensional random barriers model under the condition that the jumps are made only to neighboring sites, the Laplace transform of equation (2.1) becomes $\left(G_{n}=F_{n}\right.$ in this case)

$$
\left[s+p U_{n}\right] G_{n}-G_{n}^{0}=\omega_{n+1 / 2}\left[G_{n+1}-G_{n}\right]+\omega_{n-1 / 2}\left[G_{n-1}-G_{n}\right] .
$$

In matrix form, this can be written as

$$
\hat{V}^{1} G-G^{0}=\hat{V}^{2} G
$$

where $\hat{V}^{1}$ is diagonal matrix with the components $V_{n, n}^{1}=s+p U_{n}, V_{n, m}^{1}=0$ for $n \neq m ; \hat{V}^{2}$ is symmetrical tridiagonal matrix with the components $V_{n, n}^{2}=-\omega_{n+1 / 2}-\omega_{n-1 / 2}, V_{n, n+1}^{2}=V_{n+1, n}^{2}=\omega_{n+1 / 2}, \quad V_{n, n+m}^{2}=0$ for $m>1$. We can write formal solution of equation (3.2) as:

$$
G=\left(\hat{V}^{1}-\hat{V}^{2}\right)^{-1} G^{0}
$$

This solution is valid for each configuration, i.e. for each set of parameters $\omega_{n}$. As a result of averaging, we obtain

$$
\langle G\rangle=\left\langle\left(\hat{V}^{1}-\hat{V}^{2}\right)^{-1}\right\rangle G^{0}
$$

This relationship implies that the averaged function $\langle G\rangle$ satisfies the equation

$$
\hat{V}^{1}\langle G\rangle-G^{0}=\hat{V}^{3}\langle G\rangle,
$$

where

$$
\hat{V}^{3}=\hat{V}^{1}-\left\langle\left(\hat{V}^{1}-\hat{V}^{2}\right)^{-1}\right\rangle^{-1}
$$

The matrix $\hat{V}^{3}$ is symmetrical because the matrices $\hat{V}^{1}$ and $\hat{V}^{2}$ are symmetrical and the operation of averaging and the operation of taking an inverse matrix both retain symmetry. Although this will not be a tridiagonal matrix but it can be approximated by a tridiagonal matrix with good accuracy [12]. Therefore, we can write (3.5) as

$$
\left[s+p U_{n}\right]\langle G\rangle_{n}-G_{n}^{0}=V_{n, n+1}^{9}\left[\langle G\rangle_{n+1}-\langle G\rangle_{n}\right]+V_{n, n-1}^{9}\left[\langle G\rangle_{n-1}-\langle G\rangle_{n}\right] .
$$


The component $V_{n, n+1}^{3}$ must be symmetric function of $s+p U_{n}$ and $s+p U_{n+1}$. If $U_{n}=0$, it should be reduced to the function $\Lambda(s)$, and if $U_{n}=1$, it must be equal to $\Lambda(s+p)$. The only function satisfying these conditions is $V_{n, n+1}^{3}=\Lambda\left(\left[s+p U_{n}+s+p U_{n+1}\right] / 2\right)$ We denote it by $\Lambda_{n+1 / 2}$.

Suppose that the function $U(x)$ has a discontinuity at some point between the sites $l$ and $l-1$. Then (3.7) for the sites $l$ and $l+1$ can be written as

$$
\begin{aligned}
& {\left[s+p U_{l}\right] G\left(x_{l}, p, s\right)-G^{0}\left(x_{l}\right)=} \\
& =a \Lambda_{l+1 / 2} \frac{\partial}{\partial x} G(x, p, s)_{x=x_{l+1 / 2}}+\Lambda_{l-1 / 2}\left[G\left(x_{l-1}, p, s\right)-G\left(x_{l}, p, s\right)\right]+o[a],
\end{aligned}
$$

and

$$
\left[s+p U_{l+1}\right] G\left(x_{l+1}, p, s\right)-G^{0}\left(x_{l+1}\right)=a^{2} \frac{\partial}{\partial x}\left[\Lambda(s+p U(x)) \frac{\partial}{\partial x} G(x, p, s)\right]_{x=x_{l+1}}+o\left[a^{2}\right]
$$

where $G(x, p, s)$ is the continuous extension of the discrete function $\langle G\rangle_{n}$ : $G\left(x_{n}, p, s\right)=\langle G\rangle_{n} / a$ and $a$ is the lattice constant. The symbol $o[f(a)]$ denote a function of higher degree of smallness than $f(a)$ as $a$ tends to 0. The left side of (3.8), in view of(3.9) can be rewritten as

$$
\begin{aligned}
& {\left[s+p U_{l}\right] G\left(x_{l}, p, s\right)-G^{0}\left(x_{l}\right)=} \\
& =\left[s+p U_{l+1}\right] G\left(x_{l+1}, p, s\right)-G^{0}\left(x_{l+1}\right)+o\left[\left\{s+p U_{l+1}\right\} G\left(x_{l+1}, p, s\right)-G^{0}\left(x_{l+1}\right)\right]= \\
& =a^{2} \frac{\partial}{\partial x}\left[\Lambda(s+p U(x)) \frac{\partial}{\partial x} G(x, p, s)\right]_{x=x_{l+1}}+o\left[a^{2}\right] .
\end{aligned}
$$

(Here, the first equality holds if the initial condition $G^{0}(x)$ is smooth. We can use this assumption, since the diffusion process at large times does not depend on the exact form of the initial condition. This assumption corresponds to the assumption in real time domain that the time derivative of $G(x, p, s)$ is smooth function of $x$.) Substituting relation (3.10) into (3.8), we find

$$
\Lambda_{l-1 / 2}\left[G\left(x_{l-1}, p, s\right)-G\left(x_{l}, p, s\right)\right]=-a \Lambda_{l+1 / 2} \frac{\partial}{\partial x} G(x, p, s)_{x=x_{l+1 / 2}}+o[a] .
$$

In the continuum limit, this relation reduced to the boundary condition

$$
[G(x,, p, s)]_{+}=[G(x,, p, s)]_{-} .
$$

Here, the sign "+" denote the value of function on one side of the boundary, and the sign "-" on the other. Considering the sites to the left of discontinuity, we obtain the relation

$$
\Lambda_{l-1 / 2}\left[G\left(x_{l-1}, p, s\right)-G\left(x_{l}, p, s\right)\right]=-a \Lambda_{l-3 / 2} \frac{\partial}{\partial x} G(x, p, s)_{x=x_{l-3 / 2}}+o[a]
$$

instead of (3.11). Eliminating the expression $\Lambda_{l-1 / 2}\left[G\left(x_{l-1, p, s}\right)-G\left(x_{l, p, s}\right)\right]$ from (3.11) and (3.13) and passing to the continuum limit, we obtain the second boundary condition as

$$
\left[\Lambda(s+p U(x)) \frac{\partial}{\partial x} G(x, p, s)\right]_{+}=\left[\Lambda(s+p U(x)) \frac{\partial}{\partial x} G(x, p, s)\right]_{-} .
$$


Now consider the case where there are traps, but the particle is in the transport state at the initial time. Making the Laplace transforms of equations (2.1) and (2.2), excluding the probability of finding the particle in the traps $C_{n}$, and averaging over the parameters $\kappa_{n}$ and $v_{n}$, we obtain the equation for the probability of finding the particle in transport states as:

$$
\left[s+p U_{n}+\Sigma\left(s+p U_{n}\right)\right] F_{n}-F_{n}^{0}=\omega_{n+1 / 2}\left[F_{n+1}-F_{n}\right]+\omega_{n-1 / 2}\left[F_{n-1}-F_{n}\right] .
$$

This equation differs from (3.1) only by the presence of a configuration independent term $\sum\left(s+p U_{n}\right)$; so to this equation the above reasoning can be applied. Therefore, the function $F(x, p, s)$ satisfies the boundary conditions

$$
\begin{aligned}
{[F(x,, p, s)]_{+} } & =[F(x,, p, s)]_{-} \\
{\left[\Psi(s+p U(x)) \frac{\partial}{\partial x} F(x, p, s)\right]_{+} } & =\left[\Psi(s+p U(x)) \frac{\partial}{\partial x} F(x, p, s)\right]_{-},
\end{aligned}
$$

To obtain the boundary conditions for the function $G(x, p, s)$, it is necessary to use the relation similar to the relation (2.9):

$$
G(x, p, s)=[1+\Sigma(s+p U(x)) /(s+p U(x))] F(x, p, s) .
$$

As a result, we obtain the following boundary conditions:

$$
\begin{gathered}
{[\Phi(s+p U(x)) G(x, p, s)]_{+}=[\Phi(s+p U(x)) G(x, p, s)]_{-} .} \\
{\left[\Psi(s+p U(x)) \frac{\partial}{\partial x}\{\Phi(s+p U(x)) G(x, p, s)\}\right]_{+}=} \\
=\left[\Psi(s+p U(x)) \frac{\partial}{\partial x}\{\Phi(s+p U(x)) G(x, p, s)\}\right]_{-},
\end{gathered}
$$

If the particle is not necessarily in the transport state at the initial time, then, arguing as in subsection (2.2), we obtain the relation between functions $G(x, p, s)$ and $F(x, p, s)$

$$
G(x, p, s)=[1+\Sigma(s+p U(x)) /(s+p U(x))] F(x, p, s)+\sum_{i=1}^{N} \frac{\theta_{i}^{0}(x)}{s+p U(x)+\nu_{i}} .
$$

The boundary conditions for the function $F(x, p, s)$ remain unchanged. Substituting the relation (3.21) in (3.16) and (3.17), we find the boundary conditions for the function $G(x, p, s)$

$$
\begin{gathered}
{[\Phi(s+p U(x)) G(x, p, s)-\Omega(s+p U(x))]_{+}=} \\
=[\Phi(s+p U(x)) G(x, p, s)-\Omega(s+p U(x))]_{-} \cdot \\
{\left[\Psi(s+p U(x)) \frac{\partial}{\partial x}\{\Phi(s+p U(x)) G(x, p, s)-\Omega(s+p U(x))\}\right]_{+}=} \\
=\left[\Psi(s+p U(x)) \frac{\partial}{\partial x}\{\Phi(s+p U(x)) G(x, p, s)-\Omega(s+p U(x))\}\right]_{-} .
\end{gathered}
$$

If at the initial time the probability is concentrated at one point and this point is not on the boundary, the last boundary conditions are reduced to the boundary conditions (3.19), and (3/20). Henceforth, we will consider only such case. 


\section{Backward equation}

In this section, we show that the distribution of the functional $H\left(x_{0}, A, t\right)$ can be found not only by integrating the joint distribution $G\left(x, p, s ; x_{0}\right)$, but also by solving the backward equation.

First consider the case when, at the initial time, the particle is in the transport state. In this case, $\phi(z)=1$ and $G\left(x, p, s ; x_{0}\right)=G_{r}\left(x, p, s ; x_{0}\right)$. We assert that the distribution of the functional (1.1) corresponding to the initial position of the particle $x$ in the Laplace space $(A \rightarrow p, t \rightarrow s)$ satisfies the backward equation

$$
[s+p U(x)] f(x, p . s)-1=a^{2} \Phi(s+p U(x)) \frac{\partial}{\partial x}\left\{\Psi(s+p U(x)) \frac{\partial}{\partial x} f(x, p . s)\right\}
$$

and the backward boundary conditions

$$
\begin{gathered}
{[f(x, p, s)]_{+}=[f(x, p, s)]_{-} .} \\
{\left[\Psi(s+p U(x)) \frac{\partial}{\partial x} f(x, p, s)\right]_{+}=\left[\Psi(s+p U(x)) \frac{\partial}{\partial x} f(x, p, s)\right]_{-} .}
\end{gathered}
$$

(A similar problem with a discontinuity across an interior boundary was considered in [16].) In order to prove this assertion, we multiply (2.18) by $f(x, p, s)$ and integrate with respect to $x$. On the left side, we obtain

$$
\int_{-\infty}^{\infty}(s+p U(x)) f(x, p, s) G\left(x, p, s ; x_{0}\right) \mathrm{d} x-f\left(x_{0}, p, s\right) .
$$

The right-hand side, after a double integration by parts, can be written as

$$
\begin{aligned}
& a^{2} \int_{-\infty}^{\infty} G\left(x, p, s ; x_{0}\right) \Phi(s+p U(x)) \frac{\partial}{\partial x}\left\{\Psi(s+p U(x)) \frac{\partial}{\partial x} f(x, p . s)\right\} \mathrm{d} x+ \\
& +a^{2} \sum \pm\left\{f(x, p, s) \Psi(s+p U(x)) \frac{\partial}{\partial x}\left\{\Phi(s+p U(x)) G\left(x, p . s ; x_{0}\right)\right\}-\right. \\
& \left.-\Phi(s+p U(x)) G\left(x, p, s ; x_{0}\right) \Psi(s+p U(x)) \frac{\partial}{\partial x} f(x, p . s)\right\}_{ \pm} .
\end{aligned}
$$

Here the sum is taken over all points of discontinuity. Each point of discontinuity gives two terms of the sum with opposite signs. In view of the boundary conditions $(3.19,3.20)$ and $(4.2,4.3)$, the sum vanishes. The integral term in view of (4.1) can be rewritten in the form

$$
f\left(x_{0}, p, s\right)=\int_{-\infty}^{\infty} G\left(x, p, s ; x_{0}\right) \mathrm{d} x .
$$

Equation (4.6) and (4.4), we find

$$
f\left(x_{0}, p, s\right)=\int_{-\infty}^{\infty} G\left(x, p, s ; x_{0}\right) \mathrm{d} x .
$$

Thus, if a function $f(x, A, t)$ satisfies the equation (4.1) and the boundary conditions $(4.2,4.3)$, then this function is the Laplace transform of the distribution of the functional (1.1) corresponding to the initial position of the particle $x: f(x, A, t)=H(x, A, t)$.

Now consider the case when, at the initial time, the particle is not necessarily in the transport state. From equation $(2.18)$ and the boundary conditions $(3.19,3.20)$, it follows that 
the function $G_{r}\left(x, p, s ; x_{0}\right)$ corresponding to the function $\phi(z)$ different from unity, and the function $G_{r}\left(x, p, s ; x_{0}\right)$ corresponding to the function $\phi(z)$ equal to unity (call it $\left.G_{r}\left(x, p, s ; x_{0}\right)\right)$, are connected by the relation

$$
G_{r}\left(x, p, s ; x_{0}\right)=\phi(s+p U(x)) G_{r}^{0}\left(x, p, s ; x_{0}\right) .
$$

Substituting this relation into (2.27) and integrating with respect to $x$, we obtain

$$
H\left(x_{0}, p, s\right)=\frac{1}{s+p U\left(x_{0}\right)}+\phi\left(s+p U\left(x_{0}\right)\right)\left[H^{0}\left(x_{0}, p, s\right)-\frac{1}{s+p U\left(x_{0}\right)}\right],
$$

where $H^{0}\left(x_{0}, p, s\right)$ is the distribution of the functional corresponding to the function $\phi(z)$ equal to unity. Thus, in this case, it is necessary to solve equation (4.1) with the boundary conditions (4.2 and 4.3) and then substitute the functions found in the relation (4.9).

\section{The residence time in the half-space}

Consider the problem of calculating the distribution of the functional (1.1) with the function $U(x)$ equal to unity at $x>0$ and zero at $x<0$.

Equation (4.1), in this case, can be written as

$$
s H^{0}\left(x_{0}, p, s\right)-1=\left\{\begin{array}{l}
a^{2} \Theta(s) \frac{\partial^{2}}{\partial x_{0}^{2}} H^{0}\left(x_{0}, p, s\right), x_{0}<0 \\
a^{2} \Theta(s+p) \frac{\partial^{2}}{\partial x_{0}^{2}} H^{0}\left(x_{0}, p, s\right)-p H^{0}\left(x_{0}, p, s\right), x_{0}>0 .
\end{array}\right.
$$

The solution of this equation is the form $[4,22]$

$$
H^{0}\left(x_{0}, p, s\right)=\left\{\begin{array}{l}
C_{1} \exp \left[\frac{x_{0}}{a} \sqrt{\frac{s}{\Theta(s)}}\right]+\frac{1}{s}, x_{0}<0 \\
C_{2} \exp \left[-\frac{x_{0}}{a} \sqrt{\frac{s+p}{\Theta(s+p)}}\right]+\frac{1}{s+p}, x_{0}>0 .
\end{array}\right.
$$

Constant $C_{1}$ and $C_{2}$ are determined from the boundary condition (4.2 and 4.3)

$$
\begin{aligned}
& C_{2}+\frac{1}{s+p}=C_{1}+\frac{1}{s}, \\
& \Xi(s+p) C_{2}=-\Xi(s) C_{1},
\end{aligned}
$$

where $\Xi(s)=\psi(s) \sqrt{s /\left(a^{2} \Theta(s)\right)}$. Substituting the resulting function $H^{0}\left(x_{0}, p, s\right)$ into formula (4.9) we obtain the final solution

$$
H\left(x_{0}, p, s\right)=\left\{\begin{array}{l}
\frac{1}{s}-\frac{\phi(s) p}{s(s+p)} \frac{\Xi(s+p)}{\Xi(s+p)+\Xi(s)} \exp \left[\frac{x_{0}}{a} \sqrt{\frac{s}{\Theta(s)}}\right], x_{0}<0 \\
\frac{1}{s+p}+\frac{\phi(s+p) p}{s(s+p)} \frac{\Xi(s)}{\Xi(s+p)+\Xi(s)} \exp \left[-\frac{x_{0}}{a} \sqrt{\frac{s+p}{\Theta(s+p)}}\right], x_{0}>0 .
\end{array}\right.
$$

With the forward equation the calculation will be more complex. It is necessary to consider the cases $x_{0}<0$ and $x_{0}>0$. If $x_{0}<0$ then, according to the formula (2.17), the function $G\left(x, p, s ; x_{0}\right)$ can be represented as 


$$
G\left(x, p, t ; x_{0}\right)=G_{r}\left(x, p, s ; x_{0}\right)+\frac{1-\phi(s)}{s} \delta\left(x-x_{0}\right) .
$$

In the regions $x_{0}<0$ and $x_{0}>0$, the function $G_{r}\left(x, p, s ; x_{0}\right)$ satisfies the equations

$$
s G_{r}\left(x, p, s ; x_{0}\right)-\phi(s) \delta\left(x-x_{0}\right)=a^{2} \Theta(s) \frac{\partial^{2}}{\partial x^{2}} G_{r}\left(x, p, s ; x_{0}\right)
$$

and

$$
(s+p) G_{r}\left(x, p, s ; x_{0}\right)=a^{2} \Theta(s+p) \frac{\partial^{2}}{\partial x^{2}} G_{r}\left(x, p, s ; x_{0}\right),
$$

respectively. A bounded at infinity solution of these equations has the form

$$
G_{r}\left(x, p, s ; x_{0}\right)=\left\{\begin{array}{l}
C_{1} \exp \left[\frac{x}{a} \sqrt{\frac{s}{\Theta(s)}}\right], x<x_{0} \\
C_{2} \exp \left[-\frac{x}{a} \sqrt{\frac{s+p}{\Theta(s+p)}}\right], x>0 \\
C_{3} \exp \left[\frac{x}{a} \sqrt{\frac{s}{\Theta(s)}}\right]+C_{4} \exp \left[-\frac{x}{a} \sqrt{\frac{s}{\Theta(s)}}\right], x_{0}<x<0 .
\end{array}\right.
$$

At point $x=0$, this solution must satisfy the boundary conditions (3.19 and 3.20)

$$
\begin{gathered}
\Phi(s)\left(C_{3}+C_{4}\right)=\Phi(s+p) C_{2}, \\
\Phi(s) \Xi(s)\left(C_{3}-C_{4}\right)=\Phi(s+p) \Xi(s+p) C_{2} .
\end{gathered}
$$

At point $x=x_{0}$, the function $G\left(x, p, s ; x_{0}\right)$ must be continuous, and its first derivative should have a jump caused by the delta-function:

$$
\begin{gathered}
C_{1} \exp \left[\frac{x_{0}}{a} \sqrt{\frac{s}{\Theta(s)}}\right]=C_{3} \exp \left[\frac{x_{0}}{a} \sqrt{\frac{s}{\Theta(s)}}\right]+C_{4} \exp \left[-\frac{x_{0}}{a} \sqrt{\frac{s}{\Theta(s)}}\right], \\
a^{2} \Phi(s) \Xi(s)\left\{C_{3} \exp \left[\frac{x_{0}}{a} \sqrt{\frac{s}{\Theta(s)}}\right]-C_{4} \exp \left[-\frac{x_{0}}{a} \sqrt{\frac{s}{\Theta(s)}}\right]-C_{1} \exp \left[\frac{x_{0}}{a} \sqrt{\frac{s}{\Theta(s)}}\right]\right\}=-\phi(s) .
\end{gathered}
$$

The distribution $H\left(x_{0}, p, s\right)$ is calculated by the formula (2.6)

$$
\begin{aligned}
& H\left(x_{0}, p, s\right)=\frac{1-\phi(s)}{s}+C_{1} \sqrt{\frac{a^{2} \Theta(s)}{s}} \exp \left[\frac{x_{0}}{a} \sqrt{\frac{s}{\Theta(s)}}\right]+C_{2} \sqrt{\frac{a^{2} \Theta(s+p)}{s+p}}+ \\
& +C_{3} \sqrt{\frac{a^{2} \Theta(s)}{s}}\left\{1-\exp \left[\frac{x_{0}}{a} \sqrt{\frac{s}{\Theta(s)}}\right]\right\}+C_{4} \sqrt{\frac{a^{2} \Theta(s)}{s}}\left\{\exp \left[-\frac{x_{0}}{a} \sqrt{\frac{s}{\Theta(s)}}\right]-1\right\} .
\end{aligned}
$$

Finding constant $C_{1}, C_{2} C_{3} C_{4}$, and substituting them into the last formula, we get a result identical to the result obtained with the backward equation. Case $x_{0}>0$ is similar.

In $[4,22]$, the authors obtained a solution of this problem for the special case when: there are no barriers, $\psi(s)=$ const; the particle at the initial time is in the transport state, $\phi(z)=1$; and the function $\Phi(s)$ corresponds to the anomalous subdiffusion, $\Phi(s) \sim s^{1-\alpha}$. In this particular case, provided $x_{0}=0$, we get the following distribution:

$$
H(0, p, s)=\frac{s^{\alpha / 2-1}+(s+p)^{\alpha / 2-1}}{s^{\alpha / 2}+(s+p)^{\alpha / 2}} .
$$


Having performed the inverse Laplace transform, the authors obtained the following distribution for the relative residence time in the half-space $p_{+}=A / t$ for the $t \rightarrow \infty$ :

$$
f\left(p_{+}\right)=\frac{\sin (\pi \alpha / 2)}{\pi} \frac{p_{+}^{\alpha / 2-1}+\left(1-p_{+}\right)^{\alpha / 2-1}}{p_{+}^{\alpha / 2}+\left(1-p_{+}\right)^{\alpha / 2}+2 \cos (\pi \alpha / 2) p_{+}^{\alpha / 2}\left(1-p_{+}\right)^{\alpha / 2}} .
$$

In the limit $\alpha \rightarrow 0$, this distribution takes the form of two delta-peaks located at $p_{+}=0$ and $p_{+}=1$; that is, the particle is localized in one of the two half-spaces.

Distribution (5.14) is obtained under the assumption that the anomalous subdiffusion is caused by traps. What if anomalous subdiffusion is caused by barriers? We put $\Phi(s)=$ const $; \phi(z)=1 ; \psi(s) \sim s^{1-\alpha}$ in (5.5). Instead of (5.15), we find

$$
H(0, p, s)=\frac{s^{-\alpha / 2}+(s+p)^{-\alpha / 2}}{s^{1-\alpha / 2}+(s+p)^{1-\alpha / 2}} .
$$

and instead of (5.16) we find

$$
f\left(p_{+}\right)=\frac{\sin (\pi-\pi \alpha / 2)}{\pi} \frac{p_{+}^{-\alpha / 2}+\left(1-p_{+}\right)^{-\alpha / 2}}{p_{+}^{2-\alpha}+\left(1-p_{+}\right)^{2-\alpha}+2 \cos (\pi-\pi \alpha / 2) p_{+}^{1-\alpha / 2}\left(1-p_{+}\right)^{1-\alpha / 2}} .
$$

For small $\alpha$ we have $\sin (\pi-\pi \alpha / 2) \approx \pi \alpha / 2, \cos (\pi-\pi \alpha / 2) \approx-1+(\pi \alpha / 2)^{2} / 2$,

$$
f\left(p_{+}\right) \approx \frac{\alpha}{2} \frac{p_{+}^{-\alpha / 2}+\left(1-p_{+}\right)^{-\alpha / 2}}{\left[p_{+}^{1-\alpha / 2}-\left(1-p_{+}\right)^{1-\alpha / 2}\right]^{2}+(\pi \alpha / 2)^{2} p_{+}^{1-\alpha / 2}\left(1-p_{+}\right)^{1-\alpha / 2}} .
$$

We can see that, in the limit $\alpha \rightarrow 0$, the distribution (5.18) takes the form of a deltapeak located at $p_{+}=1 / 2$; that is, the time spent in the area $x>0$ is equal to half of the total time. Disagreement with the previous result is explained by the fact that subdiffusion caused by barriers is stationary. The mobility of a particle does not change with time [10]. For small $\alpha$, the particle cannot move away from the initial position at a large distance because the labyrinth is too tangled (or the environment is too crowded). But in the space available to it, the particle continues to move, spending approximately equal time in both areas $x>0$ and $x<0$.

\section{Conclusions.}

In the present paper, both forward and backward Feynman-Kac equations for random walks in disordered media are obtained. As a model of a disordered medium, the Schirmacher model, which is the combination of the random barriers model and the multiple-trapping model, is used. This model takes into account the presence in real disordered media both traps and different kinds of obstacles. Through this it can describe both non-stationary subdiffusion due to a delay of particles in traps and stationary subdiffusion due to the negative velocity correlations. It is shown that the distributions of functionals for media with different microscopic structures may differ significantly. This means that the obtained equations can be used to determine the structure of a disordered medium. For this purpose the parameters of these equations, in particular, memory functions $\Lambda(t)$ and $\Sigma(t)$ must be chosen so that the theoretical distribution of functional coincide with the experimental one. Having these memory functions, and knowing the dependence of these functions on the parameters characterizing the structure of the medium (see $[7,15,18]$ ), it is possible, in principle, to find these medium structure parameters. 


\section{References}

1. N. Agmon. Residence times in diffusion processes // J. Chem. Phys. - 1984. -V. 81. P. 3644-3647.

2. Bar-Haim, J. Klafter On mean residence and first passage times in finite onedimensional systems // J. Chem. Phys. - 1998. - V. 109. - P. 5187-5193.

3. Cairoli, A.Baule Anomalous processes with general waiting times: functionals and multi-point structures // arXiv:1411.7005[cond-mat.stat-mech].

4. S. Carmi, L. Turgeman, E. Barkai On distributions of functionals of anomalous diffusion paths: a fractional Feynman- Kac approach // J. Stat. Phys. - 2010. - V. 141. - P. 1071-1092.

5. G. Foltin, K. Oerding, Z. Racz, R.L. Workman, R.P.K.Zia Width distribution for random-walk interfaces // Phys. Rev. E - 1994. - V. 50. - R639.

6. A.H. Gandjbakhche, G.H. Weiss Descriptive parameter for photon trajectories in a turbid medium // Phys. Rev. E - 2000. - V. 61. - P. 6958-6970.

7. K. Godzik, W. Schirmacher Theory of dispersive transport in amorphous semiconductors // J. de Phys. (Paris). - 1981. - V. 42. - P. 127-131.

8. D.S. Grebenkov Residence times and other functionals of reflected Brownian motion // Phys. Rev. E - 2007. - V. 76. - P. 041139.

9. D.S. Grebenkov NMR survey of reflected Brownian motion // Rev. Mod. Phys. 2007. V. 79. - P. 1077-1137.

10. R.L. Jack, P. Sollich Duality symmetries and effective dynamics in disordered hopping models // J. Stat. Mech.: Theory and Experiment. - 2009. - P. 11011.

11. M. Kac On distributions of certain Wiener functionals // Trans. Am. Math. Soc. 1949. - V. 65. - P. 1-13.

12. V.M. Kenkre, Z. Kalay, P.E. Parris Extensions of effective-medium theory of transport in disordered systems // Phys. Rev. E - 2009. - V. 79. - P. 011114.

13. S.N. Majumdar, A. Comtet Local and occupation time of a particle diffusing in a random medium // Phys. Rev. Lett. - 2002. - V. 89. - P. 060601.

14. S.N. Majumdar Brownian functionals in physics and computer science // Curr. Sci. - 2005. - V. 89. - P. 2076.

15. Movaghar, M. Grunewald, B. Pohlmann, et al. Theory of hopping and multipletrapping in disordered systems // J. Stat. Phys. - 1983. - V. 30. - P. 315-334.

16. Ovaskainen, S.J. Cornell. Biased movement at a boundary and conditional occupancy times for diffusion processes // J. Appl. Prob. - 2003. - V. 40. - P. 557580 .

17. S. Sabhapandit, S.N. Majumdar, A. Comtet Statistical properties of functionals of the paths of a particle diffusing in a one-dimensional random potential // Phys. Rev. E - 2006. - V. 73. - P. 051102.

18. W. Schirmacher Microscopic theory of dispersive transport in disordered semiconductors // Sol. State Comm. - 1981. - V. 39. - P. 893-897.

19. V.P. Shkilev Equations for the distributions of functionals of a random-walk trajectory in an inhomogeneous medium // J. Exp. Theor. Phys. - 2012. - V. 114. P. 172-181.

20. V.P. Shkilev Boundary conditions for the subdiffusion equation // J. Exp. Theor. Phys. - 2013. - V. 116. -P. 703-710.

21. V.P. Shkilev Subdiffusion of mixed origin with chemical reactions // J. Exp. Theor. Phys. - 2013. - V. 117. - P. 1066-1070.

22. L. Turgeman, S. Carmi, E. Barkai Fractional feynman-kac equation for nonbrownian functionals // Phys. Rev. Lett. - 2009. - V. 103. - P. 190201. 


\title{
ФУНКЦІЯ РОЗПОДІЛУ ТРАЕКТОРІЇ ЧАСТИНКИ, ЯКА ЗДІЙСНЮЄ ВИПАДКОВІ БЛУКАННЯ В НЕВПОРЯДКОВАНОМУ СЕРЕДОВИЩІ
}

\author{
В.П. Шкілев, В.В. Лобанов
}

Інститут хімії поверхні ім. О.О. Чуйка Національної академії наук України вул. Генерала Наумова, 17, Київ, 03164, Україна, lobanov@isc.gov.иа

Розв'язана задача про знаходження функиії розподілу траєкторії частинки, щзо здійснює випадкові блукання в невпорядкованому середовищі, яке містить як пастки, так $i$ бар'єри. В якості моделі невпорядкованого середовища використана модель Ширмахера, яка є комбінацією моделей випадкових бар'єрів $i$ багаторазового захоплення частинки. Сформульовано прямі і зворотні рівняння Фейнмана-Каца $з$ граничними умовами в точках розриву. Як приклад отримано розподіл часу перебування частинки в півпросторі. Показано, щзо різні типи аномальної субдифузії, обумовленої пастками і бар'єрами, дають функиії розподілу, які сильно розрізняються.

\section{ФУНКЦИЯ РАСПРЕДЕЛЕНИЯ ТРАЕКТОРИИ ЧАСТИЦЫ, СОВЕРШАЮЩЕЙ СЛУЧАЙНОЕ БЛУЖДАНИЕ В НЕУПОРЯДОЧЕННОЙ СРЕДЕ}

\section{В.П. Шкилев, В.В. Лобанов}

Институт химии поверхности им. А.А. Чуйко Наџиональной академии наук Украиньл ул. Генерала Наумова, 17, Киев, 03164, Украина, lobanov@isc.gov.иa

Решена задача о нахождении функиии распределения траектории частиџы, совершающей случайное блуждание в неупорядоченной среде, которая содержит как ловушки, так и барьеры. В качестве модели неупорядоченной среды использована модель Ширмахера, которая представляет собой комбинаџию моделей случайных барьеров и многократного захвата частицы. Сформулированы прямые и обратные уравнения Фейнмана-Кача с граничными условиями в точках разрыва. В качестве примера получено распределение времени пребывания частищы в полупространстве. Показано, что различные типы аномальной субдиффузии, обусловленной ловушками и барьерами, дают сильно различающиеся функции распределения. 